\title{
BMJ Open Effects of placebos without deception compared with no treatment: protocol for a systematic review and meta-analysis
}

\author{
Grace Petkovic, ${ }^{1}$ James E G Charlesworth, ${ }^{1}$ John Kelley, ${ }^{2,3}$ Franklin Miller, ${ }^{4}$ \\ Nia Roberts, ${ }^{1}$ Jeremy Howick ${ }^{1}$
}

To cite: Petkovic G, Charlesworth JEG, Kelley J, et al. Effects of placebos without deception compared with no treatment: protocol for a systematic review and meta-analysis. BMJ Open 2015;5:e009428. doi:10.1136/ bmjopen-2015-009428

- Prepublication history and additional material is available. To view please visit the journal (http://dx.doi.org/ 10.1136/bmjopen-2015009428).

GP and JEGC contributed equally.

Received 17 July 2015 Revised 15 September 2015 Accepted 7 October 2015

CrossMark

For numbered affiliations see end of article.

Correspondence to Grace Petkovic; grace. petkovic@hmc.ox.ac.uk

\section{ABSTRACT}

Introduction: Placebos have long provided a robust control for evaluating active pharmacological preparations, but frequently demonstrate a variable therapeutic effect when delivered in double-blinded placebo-controlled trials. Delivery of placebos as treatment alone has been considered unethical, as it has been thought that deception is essential for their effect. However, recent evidence suggests that clinical benefit can be derived from placebos delivered without deception (unblinded/open-label) manner. Here, we present a protocol for the first systematic review and meta-analysis of studies of the effects of non-deceptive placebos compared with no treatment.

Methods and analysis: This protocol will compare the effect of placebos delivered non-deceptively to no treatment. It will also assess the methods of delivery used for non-deceptive placebos. Studies will be sought through relevant database searches and will include those within disease settings and those among healthy controls. To be included, trials must include both non-deceptive (open-label) placebo and no treatment groups. All data extraction and analysis will be conducted by two independent reviewers. The analysis will evaluate any differences in outcome measures between the non-deceptive placebo and no treatment groups. Outcome measures will be the clinically-relevant outcomes detailed in the primary papers. The delivery methods, such as verbal instructions, which may provide positive expectations and outcomes, of non-deceptive placebos will also be assessed. Each study will be comprehensively assessed for bias. Subgroup analyses will identify any discrepancies among heterogeneous data.

Ethics and dissemination: This review does not require ethical approval. The completed review will be widely disseminated by publication and social media where appropriate. This protocol has been registered on PROSPERO (2015:CRD42015023347).

\section{INTRODUCTION}

Use of placebos in clinical practice is widespread $^{1}$ and may be a cost-effective option for enhancing the care of ailments such as mild pain and depression. However, recommending or administering placebo interventions is often considered unethical because doctors must, allegedly, deceive patients by concealing the nature of the placebo therapy. ${ }^{2}{ }^{3}$ While several studies have investigated non-deceptive placebos, ${ }^{4-10}$ a systematic review of these studies has not been conducted. ${ }^{11}$ Placebos are most effective for stress-related conditions, ${ }^{12}$ pain, depression, anxiety and nausea. ${ }^{13}$

It is suggested that placebos work both by inducing positive expectations and through classical conditioning. Therefore, it may be anticipated that open-label placebos, which cause the patient to expect an inert intervention, will not induce the same level of conscious expectations in a patient as a deceptive placebo. However, open-label placebos have often been combined with positive suggestions such as "we are unsure how placebos work when given honestly, but we have found that they help many people with your condition". 14

Open-label placebos, when combined with an expectation of therapeutic benefit, may therefore improve healthcare outcomes via a range of downstream mechanisms, without the ethical worries inherent in deceptive placebos. ${ }^{15}$

\section{Rationale: how open-label placebos might} work

The mechanisms of action of open-label placebos are becoming increasingly well understood, but are currently speculative. Classical conditioning, a 'bottom up' mechanism, is perhaps supported by the most evidence: the conditioned expectation of a reward has been shown to activate the brain's conditioning mechanism. ${ }^{16}$ Supporting the conditioning hypothesis, a recent study of open-label placebos for treating pain showed that the open-label placebo effect persisted in patients who had been conditioned for 
longer periods (4 days) but not shorter (1 day) durations. ${ }^{17}$ Conditioned responses can be triggered by nonconscious stimuli. ${ }^{18} 19$ The body's conditioned response to open-label placebos might not be limited to the brain's reward mechanism. Recent work on embodied cognition suggests that a person's response to open-label placebos could arise from extra-cerebral activity. ${ }^{20}$

If the open-label placebo is accompanied by a suggestion that the placebo is or might be effective, then conscious expectancy a-'top down' mechanism-could also play a role. Clinical studies have shown that open administration (where the patient knew they were receiving treatment and expected a positive outcome) of pain relief or anti-anxiety medication resulted in enhanced results across conditions such as pain, anxiety and Parkinson's disease, when compared with covert administration (where the patient did not know they were receiving treatment). ${ }^{17}$ The effects of influencing patients' expectations on physiological outcomes has been most extensively documented in the field of pain research, where an expectation of pain relief has been found to activate neurological systems involved in regulating pain such as the dopamine reward system and the endogenous opioid system. ${ }^{21}$ Conversely, negative expectations have been shown to adversely affect health, most notably by increasing pain. ${ }^{22}$

It is also possible that the social interaction with a healthcare practitioner plays a role in explaining the effects of open-label placebos. Social support-of which healthcare practitioner support could be considered a component-is a well-established determinant of health. Not only can social networks provide support in the form of care and advice, ${ }^{23}$ but social networks have also been shown to influence the neuroendocrine response. ${ }^{24}$ Since encounters with a healthcare practitioner are social events, we might expect such encounters to enhance the benefits of social networks and to mitigate the negative effects associated with a lack of social networks.

While the hypothetical mechanisms could all work independently, they are more likely to operate together, producing variable effects depending on the individual and the condition.

\section{Why it is important to do this review?}

Surveys around the world suggest $17-80 \%$ of doctors have prescribed placebos, such as saline injections, in routine practice. ${ }^{125}$ Yet placebo use during routine care is often considered unethical for several alleged reasons $^{3} 15$ 26-35 including that: ${ }^{234-44}$

1. Placebos need to be delivered deceptively (disguised as though they were 'real' treatments) to be effective. Since deception requires breach of trust and violates patient autonomy, it is arguably unethical;

2. Placebos are allegedly ineffective.

Both reasons rest on empirical claims that require further analysis. There have been numerous investigations of placebo effects. While earlier estimates were undoubtedly exaggerated, ${ }^{36} 37$ even skeptics admit that for some conditions such as pain and depression, placebos are likely to be effective. ${ }^{13} 3839$

Yet whether placebos need to be delivered deceptively in order to be effective requires further investigation. Several studies suggest that non-deceptive placebos can be effective, ${ }^{4-10}{ }^{14}$ but a systematic review is required to provide more definitive evidence.

A 2010 Cochrane Review of placebo effects for all clinical conditions included some of the studies that will be included in this review. ${ }^{13}$ However, there are three relevant differences between this review and the Cochrane review. First, the Cochrane review is not up to date (relevant studies have been conducted since). ${ }^{14}{ }^{40}$ Second, no subgroup analysis addressing our specific question about the effects of non-deceptive placebos was included. Third and most importantly, data about the 'contents' of the open-label placebos, ${ }^{41}$ and how the open-label placebos were delivered (eg, the verbal instructions accompanying the pills) were not extracted or reported in the Cochrane Review. Information about how the open-label placebos were delivered is essential for drawing out any ethical implications of the effects of open-label placebos.

\section{Aims and objectives}

1. To assess the effect of placebos delivered nondeceptively compared to no treatment.

2. To assess the delivery of open-label placebos; specifically the verbal instructions which may provide positive expectations and outcomes.

\section{METHODS}

\section{Searches}

Searching for relevant studies in this area is challenging because of the absence of a common terminology for interventions in which practitioner empathy and communication of positive expectations is manipulated. Eligible studies can be found in areas ranging from placebo research, patient-practitioner communication and psychology. Our search strategy is based on the one used by $\mathrm{Di}$ Blasi et $a t^{42}$ and Mistiaen et al (2015) with the exception that we focus exclusively on open-label placebos.

The MEDLINE search strategy is given in online supplementary appendix 1 and will be adapted as required for the databases listed below. The search strategy will consist of keywords and synonyms for the intervention, placebo without deception. Relevant thesaurus terms will be used if available.

\section{Electronic searches}

Searches, using the strategy listed in online supplementary appendix 1, will start from the start date of the database through to September 2015. We will search using CINAHL (EBSCOHost), EMBASE (OvidSP), LILACS (Virtual Health Library), OpenGREY (http://opengrey. eu), Dissertations \& Theses (Proquest), PsycINFO 
(OvidSP), MEDLINE \& MEDLINE In-process (OvidSP), Sociological Abstracts (Proquest), The Cochrane Central Register of Controlled Trials (CENTRAL, The Cochrane Library, Wiley), Science Citation Index (Web of Science, Thomson Reuters) and Consumers and Communication Review Group Specialised Register.

In addition, we will search proceedings of placebo-specific conferences and contact experts in the field and authors of included studies for advice about other studies. We will also search the online clinical trial registers ClinicalTrials.gov and International Standard Randomised Controlled Trial Number (ISRCTN).

Searches will not be restricted to those published in English. Trials reported in all languages will be included. Abstracts of articles not published in English will, where an abstract in English is unavailable, be translated using an online translator. If the abstract appears to meet the inclusion criteria, it will be referred to a professional translator service. The translated article will be sent to the study authors to confirm the accuracy of the translation and will be provided as online supplementary material in the published review (if permission of the original publisher can be obtained).

All returned records will be combined into a Reference Manager database, with duplicate records removed.

\section{Inclusion criteria}

We will include studies of placebo interventions (such as sugar pills, saline injections and sham procedures) delivered 'openly' only where there is also a 'no treatment' condition. Participants given open-label placebos must be told they are receiving a placebo whereas 'no treatment' may include people on a waiting list or those simply left untreated. We will include studies of participants with a particular medical condition (such as pain, depression or irritable bowel syndrome) or members of the general population (eg, a response to placebo alcohol delivered without deception). Examples of studies that we believe will be eligible for inclusion into this systematic review are listed in table 1. Trials comparing placebos with and without deception that lack a no treatment group will be excluded.

\section{Selection of studies}

Two authors will independently screen all titles and abstracts identified from searches to determine which meet the inclusion criteria. We will retrieve in full text any papers identified as potentially relevant by at least one author. Two review authors will independently screen full-text articles for inclusion or exclusion, with discrepancies resolved by discussion and by consulting a third author, if necessary, to reach consensus. All potentially relevant papers excluded from the review at this stage will be listed as excluded studies, with reasons provided in the 'Characteristics of excluded studies' table. We will also provide citation details and any available information about ongoing studies, and collate and report details of duplicate publications, so that each study (rather than each report) is the unit of interest in the review. We will report the screening and selection process in an adapted PRISMA flow chart. ${ }^{43}$

\section{Reporting of main outcomes}

The 'Summary of Findings' table will include no more than seven outcomes per table (including harms) and will not include duplicate outcomes (the same outcome by different measures). A separate table detailing the instructions given to inform participants that they received a placebo and other information about the intervention (eg, length of consultation) will be included.

\section{Primary outcomes}

We will select the primary outcome measures given by the study authors. If this is unclear we will select the most clinically relevant outcome and provide a rationale. If outcomes are measured over time, the time point identified as the primary outcome will be used, otherwise the most clinically relevant will be chosen and justified.

Table 1 Examples of the intervention

\begin{tabular}{|c|c|c|c|c|}
\hline Study & Modality & Main outcomes & Main findings & Randomised? \\
\hline Kaptchuk et al ${ }^{14}$ & $\begin{array}{l}\text { Placebo } \\
\text { pills }\end{array}$ & $\begin{array}{l}\text { IBS Global Improvement } \\
\text { Scales }\end{array}$ & $\begin{array}{l}\text { Open-label placebos outperform } \\
\text { untreated groups }\end{array}$ & Yes \\
\hline Kelley et $\left.a\right|^{40}$ & $\begin{array}{l}\text { Placebo } \\
\text { pills }\end{array}$ & Depression (HAM-D-17) & $\begin{array}{l}\text { Open-label placebos outperform } \\
\text { untreated groups }\end{array}$ & Yes \\
\hline Sandler and Bodfish ${ }^{10}$ & $\begin{array}{l}\text { Placebo } \\
\text { pills }\end{array}$ & ADHD & $\begin{array}{l}\text { Open-label placebos outperform } \\
\text { untreated groups }\end{array}$ & Yes \\
\hline Aulas and Rosner ${ }^{4}$ & $\begin{array}{l}\text { Placebo } \\
\text { pills }\end{array}$ & Depression & $\begin{array}{l}\text { Open-label placebos outperform } \\
\text { untreated groups }\end{array}$ & $\begin{array}{l}\text { No } \\
\text { (before/after study) }\end{array}$ \\
\hline Park and Covi ${ }^{8}$ & $\begin{array}{l}\text { Placebo } \\
\text { pills }\end{array}$ & Anxiety & $\begin{array}{l}\text { Open-label placebos outperform } \\
\text { untreated groups }\end{array}$ & $\begin{array}{l}\text { No } \\
\text { (before/after study) }\end{array}$ \\
\hline
\end{tabular}




\section{Secondary outcomes}

We will report on the following, where available: any instructions given to the patient alongside the openlabel placebo; effects on quality of life; patient satisfaction; harms; and placebo responsiveness.

\section{Data extraction and management}

Two review authors will extract data independently from included studies. Any discrepancies will be resolved by discussion until consensus is reached, or through consultation with a third author where necessary. We will develop and pilot a data extraction form using the Cochrane Consumers and Communication Review Group Data Extraction Template (available at: http:// cccrg.cochrane.org/author-resources). Data to be extracted will include the following items: study design; types of participants; description of intervention and intervention components; description of comparison group; completeness of outcome data; outcome measures; country; and funding source. All extracted data will be entered into RevMan (RevMan 2012) by one review author, and will be checked for accuracy against the data extraction sheets by a second review author working independently.

\section{Assessment of risk of bias in included studies}

We will assess and report on the methodological risk of bias of included studies in accordance with the Cochrane Handbook. ${ }^{45}$ This recommends the explicit reporting of the following individual elements for randomised control trials (RCTs): random sequence generation; allocation sequence concealment; blinding (participants, personnel, outcome assessment); completeness of outcome data, selective outcome reporting; and other sources of bias. We will consider blinding separately for different outcomes where appropriate. We will judge each item as being at high, low or unclear risk of bias as set out in the criteria provided by Higgins and Green $^{45}$ and provide a quote from the study report and a justification for our judgment for each item in the risk of bias table.

Studies will be deemed to be at the highest risk of bias if they are scored as at high or unclear risk of bias for either the sequence generation or allocation concealment domains, based on growing empirical evidence that these factors are particularly important potential sources of bias. ${ }^{45}$

In all cases, two authors will independently assess the risk of bias of included studies, with any disagreements resolved by discussion to reach consensus. We will contact study authors for additional information about the included studies, or for clarification of the study methods, as required. We will incorporate the results of the risk of bias assessment into the review through standard tables, and systematic narrative description and commentary about each of the elements. This will lead to an overall assessment of the risk of bias of included studies and a judgment about the internal validity of the review's results. For cluster-RCTs we will also assess and report the risk of bias associated with an additional domain: selective recruitment of cluster participants.

We will also report whether the interventions and control treatments were described in sufficient detail to replicate. We will investigate and report on the most relevant causal factors.

\section{Data synthesis}

Our review is designed to be heterogeneous in terms of outcome measures because empathy and expectations are likely to be effective across many different outcomes. We also anticipate heterogeneity in terms of study participants, and intervention components/modalities. If sufficient data are available, we will nevertheless conduct a random-effects meta-analysis. We will include in the meta-analysis all relevant studies, irrespective of the risk of bias. However, we will conduct a sensitivity analysis excluding studies with an unclear or high risk of bias in the random sequence generation or allocation concealment fields. We will also conduct subgroup analyses, which will include more heterogeneous groups of studies. If there is insufficient data for pooling we will present the results in narrative format.

\section{Protocol amendments}

Deviations from the protocol listed here will be identified in the final published review.

\section{Measures of treatment effect}

For dichotomous outcomes, we will analyse data based on the number of events and the number of people assessed in the intervention and comparison groups. We will use these to calculate the risk ratio (RR) and $95 \%$ CI. For continuous measures, we will analyse data based on the mean, SD and number of people assessed for both the intervention and comparison groups to calculate the standardised mean difference (SMD) and 95\% CI. If the SMD is reported without individual group data, we will use this to report the study results.

\section{Unit of analysis issues}

If cluster-RCTs are included we will check for unit-of-analysis errors. If errors are found, and sufficient information is available, we will reanalyse the data using the appropriate unit of analysis, by taking account of the intracluster correlation (ICC). We will obtain estimates of the ICC by contacting authors of included studies, or impute them using estimates from external sources. If it is not possible to obtain sufficient information to reanalyse the data we will report effect estimates and annotate ‘unit-of-analysis' error.

\section{Dealing with missing data}

We will attempt to contact study authors to obtain missing data (participant, outcome or summary data). For participant data, we will, where possible, conduct analysis on an intention-to-treat basis; otherwise data will be analysed as 
reported. We will report on the levels of loss to follow-up and assess this as a source of potential bias.

For missing outcome or summary data we will impute missing data where possible and report any assumptions in the review. We will investigate, through sensitivity analyses, the effects of any imputed data on pooled effect estimates.

\section{Assessment of heterogeneity}

We anticipate heterogeneity in terms of intervention modalities, conditions, outcome measures, patients and effects. Where studies are considered similar enough (based on consideration of populations, interventions or other factors) to allow pooling of data using meta-analysis, we will assess the degree of heterogeneity by visual inspection of forest plots and by $\chi^{2}$ test for heterogeneity. Heterogeneity will be quantified using the $\mathrm{I}^{2}$ statistic. An $\mathrm{I}^{2}$ value of $50 \%$ or more will be considered to represent substantial levels of heterogeneity, but this value will be interpreted in light of the size and direction of effects and the strength of the evidence for heterogeneity, based on the $p$ value from the $\chi^{2}$ test. ${ }^{45}$

Where we detect substantial clinical, methodological or statistical heterogeneity across included studies we will not report pooled results from meta-analysis but will instead use a narrative approach to data synthesis. In this event we will attempt to explore possible clinical or methodological reasons for this variation by grouping studies that are similar in terms of populations, intervention features, methodological features or other factors to explore differences in intervention effects.

When too few trials are included in a meta-analysis, the $\chi^{2}$ test has little power to detect heterogeneity. Therefore, a non-significant result will not necessarily be interpreted as evidence of no heterogeneity and will be interpreted with care.

\section{Assessment of meta-biases (publication bias)}

We will assess reporting bias qualitatively based on the characteristics of the included studies (eg, if only small studies show positive effects), and if information that we obtain from contacting experts and authors of studies suggests that there are relevant unpublished studies. If we identify sufficient studies (at least 10) for inclusion in the review we will construct a funnel plot to investigate small study effects, which may indicate the presence of publication bias. We will formally test for funnel plot asymmetry, with the choice of test made based on advice in Higgins and Green, ${ }^{45}$ and bearing in mind that there may be several reasons for funnel plot asymmetry when interpreting the results.

\section{Subgroup analysis and investigation of heterogeneity}

We anticipate heterogeneity in terms of intervention modalities, conditions, outcome measures, patients and effects. If there are a sufficient number of studies, we will therefore conduct rigorous subgroup analyses. To investigate whether specific subgroups of trials reported effects of empathy/expectation induction we will compare the following subgroups, with tests of interaction:

- If three or more trials investigate the same ailment (such as pain, depression or anxiety) we will analyse these ailments separately. Subjective (patient-reported) versus objective (practitioner reported) outcomes will be analysed separately.

- Trials where a specific modality (pills, injections, sham interventions) were used will be analysed in separate groups.

\section{Sensitivity analysis}

We anticipate performing the following separate sensitivity analyses:

- Exclusion of studies with a high risk or unclear risk of bias in either the random allocation schedule or allocation concealment fields;

- Continuous data: excluding studies where assumptions about SDs had to be made because they were missing.

- Trials conducted in a clinical (as opposed to laboratory) setting.

\section{'Summary of findings' table}

We will prepare a 'Summary of findings' table to present the results of meta-analysis, based on the methods described in chapter 11 of the Cochrane Handbook for Systematic Reviews of Interventions (Schünemann Available from http://www.cochrane-handbook.orga). We will present the results of meta-analysis for the major comparisons of the review, for each of the major primary outcomes, including potential harms, as outlined in the 'Types of outcome measures' section. We will provide a source and rationale for each assumed risk cited in the table(s), and will use the GRADE system to rank the quality of the evidence using the GRADEprofiler (GRADEpro) software. ${ }^{46}$ If meta-analysis is not possible, we will present results in a narrative 'Summary of findings' table format, such as that used by Chan et $_{\text {al. }}{ }^{47}$

\section{Assessing the quality of the evidence}

The GRADE system will be used to assess and report on the quality of the evidence for each outcome using the following domains: study limitations, consistency, imprecision, indirectness and publication bias. Two authors will independently assess the quality of the evidence as implemented and described in the GRADEprofiler (GRADEpro) software. ${ }^{46}$

\section{DISSEMINATION}

This protocol has been registered on PROSPERO (2015: CRD42015023347). The eventual review will be published and subsequently widely disseminated by the university and through social media platforms. 
Author affiliations

${ }^{1}$ Nuffield Department of Primary Care Health Sciences, University of Oxford, Oxford, UK

${ }^{2}$ Psychiatry Department, Massachusetts General Hospital/Harvard Medical School, Boston, Massachusetts, USA

${ }^{3}$ Psychology Department, Endicott College, Beverly, Massachusetts, USA

${ }^{4}$ Department of Bioethics, Clinical Center, National Institutes of Health, Bethesda, Maryland, USA

Contributors $\mathrm{JH}$ devised the study, drafted the protocol, will assist with data extraction and analysis and draft the results and discussion sections. GP and JEGC revised the protocol, assisted with study design and will carry out most of the data collection and analysis, and help draft final manuscript. NR designed and conducted the search strategy and provided input on the working of the manuscript. JK provided input on the protocol, statistical analysis plan and overall structure of the manuscript. FM provided input on the drafting of the protocol and rationale for the study.

\section{Competing interests None declared.}

Provenance and peer review Not commissioned; externally peer reviewed.

Open Access This is an Open Access article distributed in accordance with the Creative Commons Attribution Non Commercial (CC BY-NC 4.0) license, which permits others to distribute, remix, adapt, build upon this work noncommercially, and license their derivative works on different terms, provided the original work is properly cited and the use is non-commercial. See: http:// creativecommons.org/licenses/by-nc/4.0/

\section{REFERENCES}

1. Howick J, Bishop FL, Heneghan C, et al. Placebo use in the United Kingdom: results from a National Survey of Primary Care Practitioners. PLoS One 2013;8:e58247.

2. GMC Good Medical Practice (2006). The duties of a doctor registered with the General Medical Council. London: GMC, 2006.

3. Bostick NA, Sade R, Levine MA, et al. Placebo use in clinical practice: report of the American Medical Association Council on Ethical and Judicial Affairs. J Clin Ethics 2008;19:58-61.

4. Aulas JJ, Rosner I. [Efficacy of a non blind placebo prescription] Encephale 2003;29:68-71.

5. Bergmann JF, Chassany $\mathrm{O}$, Gandiol J, et al. A randomised clinica trial of the effect of informed consent on the analgesic activity of placebo and naproxen in cancer pain. Clin Trials Metaanal 1994;29:41-7.

6. Dahan $R$, Caulin C, Figea L, et al. Does informed consent influence therapeutic outcome? A clinical trial of the hypnotic activity of placebo in patients admitted to hospital. BMJ 1986;293:363-4.

7. Kirsch I, Weixel LJ. Double-blind versus deceptive administration of a placebo. Behav Neurosci 1988;102:319-23.

8. Park LC, Covi L. Nonblind placebo trial: an exploration of neurotic patients' responses to placebo when its inert content is disclosed. Arch Gen Psychiatry 1965;12:36-45.

9. Pollo A, Amanzio M, Casadio C, et al. Response expectancies in placebo analgesia and their clinical relevance. Pain 2001;93:77-84.

10. Sandler AD, Bodfish JW. Open-label use of placebos in the treatment of ADHD: a pilot study. Child Care Health Dev 2008;34:104-10

11. Chalmers I. The lethal consequences of failing to make full use of all relevant evidence about the effects of medical treatments: the importance of systematic reviews. In: Rothwell PM, ed. Treating individuals: from randomised trials to personalized medicine. London: The Lancet, 2007:37-58.

12. Everly GS, Lating JM. A clinical guide to the treatment of the human stress response. 2nd edn..New York, London: Kluwer Academic/ Plenum Publishers, 2002.

13. Hróbjartsson A, Gøtzsche P. Placebo interventions for all clinica conditions. Cochrane Database Syst Rev 2010;(1):CD003974.

14. Kaptchuk TJ, Friedlander E, Kelley JM, et al. Placebos without deception: a randomized controlled trial in irritable bowel syndrome. PLoS One 2010:5:e15591.

15. Foddy B. A duty to deceive: placebos in clinical practice. $A m \mathrm{~J}$ Bioeth 2009:9:4-12.

16. Benedetti F. Placebo effects: understanding the mechanisms in health and disease. Oxford: Oxford University Press, 2009.
17. Schafer SM, Colloca L, Wager TD. Conditioned placebo analgesia persists when subjects know they are receiving a placebo. J Pain 2015;16:412-20.

18. Jensen KB, Kaptchuk TJ, Chen X, et al. A neural mechanism for nonconscious activation of conditioned placebo and nocebo responses. Cereb Cortex 2015;25:3903-10.

19. Jensen KB, Kaptchuk TJ, Kirsch I, et al. Nonconscious activation of placebo and nocebo pain responses. Proc Natl Acad Sci USA 2012;109:15959-64.

20. Fuchs T, Schlimme JE. Embodiment and psychopathology: a phenomenological perspective. Curr Opin Psychiatry 2009;22:570-5.

21. Price DD, Finniss DG, Benedetti F. A comprehensive review of the placebo effect: recent advances and current thought. Annu Rev Psychol 2008;59:565-90.

22. Bingel $\mathrm{U}$, Wanigasekera $\mathrm{V}$, Wiech $\mathrm{K}$, et al. The effect of treatment expectation on drug efficacy: imaging the analgesic benefit of the opioid remifentanil. Sci Trans/ Med 2011;3:70ra14.

23. Cohen S, Janicki-Deverts D. Can we improve our physical health by altering our social networks? Perspect Psychol Sci 2009;4:375-8.

24. Ozbay F, Johnson DC, Dimoulas E, et al. Social support and resilience to stress: from neurobiology to clinical practice. Psychiatry (Edgmont) 2007;4:35-40.

25. Fässler M, Meissner K, Schneider A, et al. Frequency and circumstances of placebo use in clinical practice-a systematic review of empirical studies. BMC Med 2010;8:15.

26. Bok S. The ethics of giving placebos. Sci Am 1974;231:17-23.

27. Boozang KM. The therapeutic placebo: the case for patient deception. Fla Law Rev 2002;54:687-746.

28. Brody $\mathrm{H}$. The lie that heals: the ethics of giving placebos. Ann Intern Med 1982;97:112-8.

29. Cheyne C. Exploiting placebo effects for therapeutic benefit. Health Care Anal 2005;13:177-88

30. Foddy $B$. Response to open peer commentaries on "A duty to deceive: placebos in clinical practice". Am J Bioeth 2009;9:W1-2.

31. Kolber AJ. A limited defense of clinical placebo deception. Yale Law Policy Rev 2007;26:75-134.

32. Miller FG, Colloca L. The legitimacy of placebo treatments in clinical practice: evidence and ethics. Am J Bioeth 2009;9:39-47.

33. Miller FG, Emanuel EJ, Rosenstein DL, et al. Ethical issues concerning research in complementary and alternative medicine. JAMA 2004;291:599-604.

34. Miller FG, Wendler D, Swartzman LC. Deception in research on the placebo effect. PLoS Med 2005;2:e262.

35. Wendler D, Miller FG. Deception in the pursuit of science. Arch Intern Med 2004;164:597-600.

36. Beecher HK. The powerful placebo. J Am Med Assoc 1955;159:1602-6.

37. Kienle GS, Kiene $\mathrm{H}$. The powerful placebo effect: fact or fiction? $J$ Clin Epidemiol 1997;50:1311-8.

38. Hróbjartsson A, Gøtzsche P. Is the placebo powerless? An analysis of clinical trials comparing placebo with no treatment. $N$ Engl J Med 2001;344:1594-602.

39. Hróbjartsson A, Gøtzsche PC. Is the placebo powerless? Update of a systematic review with 52 new randomized trials comparing placebo with no treatment. J Intern Med 2004;256:91-100.

40. Kelley JM, Kaptchuk T, Cusin C, et al. Open-label placebo for major depressive disorder: a pilot randomized controlled trial. Psychother Psychosom 2012;81:312-4.

41. Research Training in the Biomedical, Behavioral, and Clinica Research Sciences. Research Training in the Biomedical, Behavioral, and Clinical Research Sciences. Washington DC, 2011.

42. Di Blasi Z, Harkness E, Ernst E, et al. Influence of context effects on health outcomes: a systematic review. Lancet 2001;357:757-62.

43. Moher D, Liberati A, Tetzlaff J, et al. Preferred reporting items for systematic reviews and meta-analyses: the PRISMA statement. BMJ 2009;339:b2535.

44. Mistiaen $P$, van Osch $M$, van Vliet $L$, et al. The effect of patientpractitioner communication on pain: a systematic review. Eur J Pain 2015. [Epub ahead of print 22 Oct 2015]

45. Higgins JJ, Green S. The Cochrane handbook for systematic reviews of interventions. Version 5.1 .0 [updated March 2011] ed. Chichester: The Cochrane Collaboration, 2011.

46. Schünemann $\mathrm{H}$, Brozek J, Oxman A, eds. GRADE handbook for grading quality of evidence and strength of recommendation. Asia Pac J Public Health, 2008.

47. Chan SP, Chui WC, Lo KW, et al. Consensus statement: appropriate consumer education and communication programs for weight-loss agents in Asia. Asia Pac J Public Health 2012;24:641-9. 